\title{
JOE: A Mobile, Inverted Pendulum
}

\author{
FELIX GRASSER \\ ALDO D'ARRIGO \\ SILVIO COLOMBI \\ Laboratory of Industrial Electronics \\ Swiss Federal Institute of Technology Lausanne \\ EPFL \\ CH-1015 Lausanne, Switzerland \\ felix.grasser@epfl.ch
}

ALFRED RUFER

\begin{abstract}
The Industrial Electronics Laboratory at the Swiss Federal Institute of Technology (EPFL) in Lausanne has built a prototype of a revolutionary two-wheeled vehicle. Due to its configuration with two coaxial wheels, each of which is coupled to a DC Motor, the vehicle is able to do stationary U-turns. A control system, made up of two decoupled state space controllers, pilots the motors so as to keep the system in equilibrium.

See http://leiwww.epfl.ch/joe for a video demonstration of JOE.
\end{abstract}

Keywords - State Space Control, Robotics, DSP, Modeling, Gyroscope.

\section{INTRODUCTION}

$\ll$ JOE» was nothing more than a crazy idea when we started simulation work two and a half years ago. The goal was to build a vehicle that could balance its driver on two coaxial wheels - a mobile, inverted pendulum.

Matlab $^{T M}$ simulations showed that it was indeed possible to control such a system using an electric motor mounted on each of the two wheels.

In order to reduce cost as well as danger for the test pilots it was decided on building a scaled down prototype carrying a weight instead of a driver.

The vehicle is composed of a chassis carrying a DC motor coupled to a planetary gearbox for each wheel, the DSP board used to implement the controller, the power amplifiers for the motors, the necessary sensors to measure the vehicle's states, the receiver for the radio control unit as well as a vertical steel bar. The batteries are bolted on the steel bar, their mass "simulating" a driver. The wheels of the vehicle are directly coupled to the output shaft of the gearboxes.

Fig. 2 shows the vehicle with its three degrees of freedom (DOF). It is able to rotate around the z-axis (pitch), a movement described by the angle $\Theta_{P}$ and the corresponding angular velocity $\omega_{P}$. The linear movement of the chassis is characterized by the position $x_{R M}$ and the speed $v_{R M}$. Additionally, the vehicle can rotate around its vertical axis (yaw) with the associated angle $\delta$ and angular velocity $\dot{\delta}$. These six state

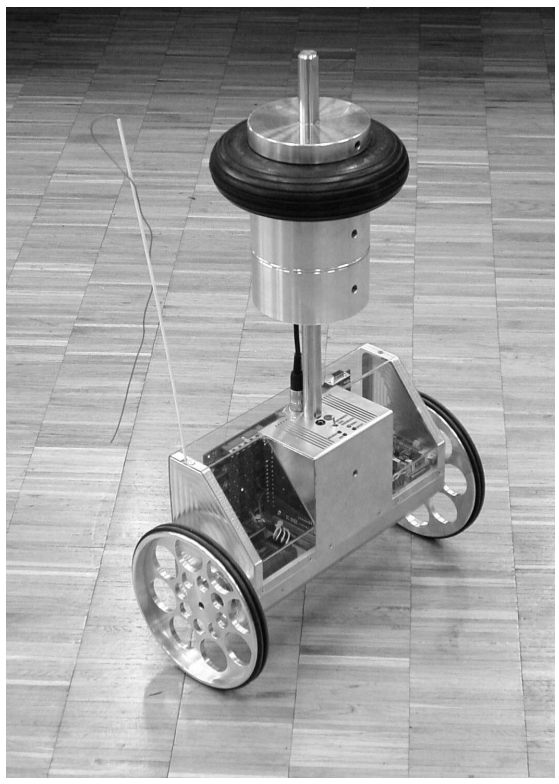

Figure 1. JOE

space variables fully describe the dynamics of the 3 DOF system.

Disturbances include forces applied to the center of gravity (CG) of the vehicle, $f_{d P}$, to the center of the left wheel, $f_{d R L}$, to the center of the right wheel, $f_{d R R}$ as well as a disturbance angle $\Theta_{d}$. $\Theta_{d}$ describes a disturbance due to a change in location of the CG. On a full size vehicle (with the driver sitting on-board) this would typically be due to the driver moving on his seat.

The vehicle is controlled by applying a torque $C_{L}$ and $C_{R}$ to the corresponding wheels.

In order to successfully control the system, the state space variables have to be quantified; either through direct measurement or an appropriate observer. Straight line position and speed as well as yaw angle and rate can be easily determined with the incremental encoders mounted on the two DC motors (provided the pitch angle and rate are known). The pitch rate is measured by a rate gyroscope. Numeri- 


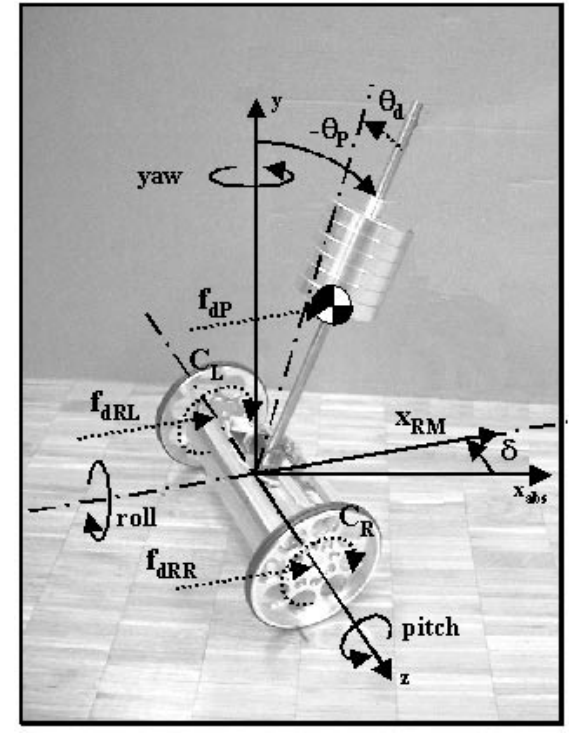

Figure 2. Definition of state space variables and disturbances.

cal integration of that signal calculates the associated pitch angle $\Theta_{P}$.

The control system is based on two decoupled state space controllers: one controlling the stability around the lateral axis (pitch) and a second one acting on the dynamics around the vertical axis (yaw). Each controller outputs a torque to be applied around its associated axis. A decoupling unit then translates these two signals into a torque to be applied to the left and right hand side motor respectively.

The driver pilots the system with a radio control unit, transmitting the desired straight line speed, $v_{R M C}$ and the desired turning rate, $\dot{\delta}_{C}$ to the on-board control system.

\section{MODELING}

In order to develop the control system, we need a dynamic model of the system that will link the system's behaviour (described by the state space variables) to its inputs (defined in section I). This model is characterized by the system's parameters (i.e. size, mass and moment of inertia of the vehicle).

A mechanical 3 DOF system can be modeled using six state space variables. For JOE, the following variables have been chosen:

$\begin{array}{lc}\mathbf{X}_{\mathbf{R M}} & \text { straight line position } \\ \mathbf{v}_{\mathbf{R M}} & \text { straight line speed } \\ \boldsymbol{\Theta}_{\mathbf{P}} & \text { pitch angle } \\ \omega_{\mathbf{P}} & \text { pitch rate } \\ \delta & \text { yaw angle } \\ \dot{\delta} & \text { yaw rate }\end{array}$

$$
\begin{array}{r}
{[\mathrm{m}]} \\
{[\mathrm{m} / \mathrm{s}]} \\
{[\mathrm{rad}]} \\
{[\mathrm{rad} / \mathrm{s}]} \\
{[\mathrm{rad}]} \\
{[\mathrm{rad} / \mathrm{s}]}
\end{array}
$$

According to the definitions in Fig. 3 the following equations of motion can be defined (we will only give the equations for the left hand wheel since the ones for

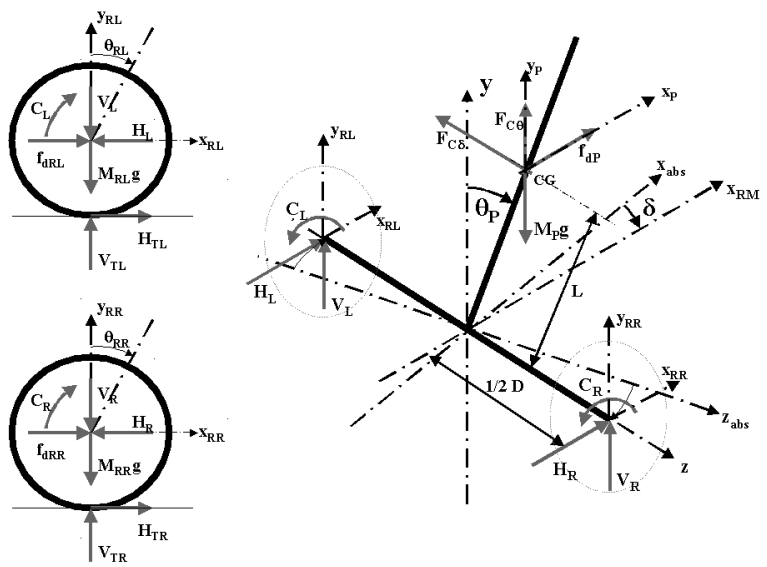

Figure 3. Free body diagram of the vehicle.

the right hand wheel are completely analogous): For the left hand wheel:

$$
\begin{gathered}
\ddot{x}_{R L} M_{R L}=f_{d R L}-H_{L}+H_{T L} \\
\ddot{y}_{R L} M_{R L}=V_{T L}-M_{R L} g-V_{L} \\
\ddot{\Theta}_{R L} J_{R L}=C_{L}-H T L R
\end{gathered}
$$

\begin{tabular}{|c|c|c|}
\hline $\mathbf{J}_{\mathbf{R L}}, \mathbf{J}_{\mathbf{R R}}$ & $\begin{array}{l}\text { Moment of inertia of the } \\
\text { rotating masses with re- } \\
\text { spect to the } z \text { axis }\end{array}$ & {$\left[\mathrm{kgm}^{2}\right]$} \\
\hline $\mathbf{M}_{\mathbf{R L}}, \mathbf{M}_{\mathbf{R R}}$ & $\begin{array}{l}\text { Mass of the rotating } \\
\text { masses connected to the } \\
\text { left and right wheel }\end{array}$ & {$[k g]$} \\
\hline $\mathbf{J}_{\mathbf{P \Theta}}$ & $\begin{array}{l}\text { Moment of inertia of the } \\
\text { chassis with respect to the } \\
z \text { axis }\end{array}$ & {$\left[\mathrm{kgm}^{2}\right]$} \\
\hline $\mathbf{J}_{\mathbf{P} \delta}$ & $\begin{array}{l}\text { Moment of inertia of the } \\
\text { chassis with respect to the } \\
y \text { axis }\end{array}$ & {$\left[\mathrm{kgm}^{2}\right]$} \\
\hline $\mathbf{M}_{\mathbf{P}}$ & Mass of the chassis & {$[k g]$} \\
\hline $\mathbf{R}$ & Radius of the wheels & {$[\mathrm{m}]$} \\
\hline D & $\begin{array}{l}\text { Lateral distance between } \\
\text { the contact patches of the } \\
\text { wheels }\end{array}$ & {$[m]$} \\
\hline $\mathbf{L}$ & $\begin{array}{l}\text { Distance between the } z \\
\text { axis and the CG of the } \\
\text { chassis }\end{array}$ & {$[m]$} \\
\hline
\end{tabular}

For the chassis:

$$
\begin{gathered}
\ddot{x}_{P} M_{P}=f_{d P}+H_{R}+H_{L} \\
\ddot{y}_{P} M_{P}=V_{R}+V_{L}-M_{P} g+F_{C \Theta} \\
\ddot{\Theta}_{P} J_{P \Theta}=\left(V_{R}+V_{L}\right) L \sin \Theta_{P} \\
-\left(H_{L}+H_{R}\right) L \cos \Theta_{P}-\left(C_{L}+C_{R}\right) \\
\ddot{\delta} J_{P \delta}=\left(H_{L}-H_{R}\right) \frac{D}{2}
\end{gathered}
$$

where: 
It has to be noted that the moment of inertia $J_{P \delta}$ in (7) depends on the angular position $\Theta_{P}$ of the chassis. However, since we shall linearize the equations for small deviations of $\Theta_{P}$ around $\Theta_{P}=0$ at a later stage, we will assume $J_{P \delta}$ to be constant at $\left.J_{P \delta}\right|_{\Theta_{P}=0}$.

We shall also assume that the wheels of the vehicle always stay in contact with the ground and that there will be no slip at the wheel's contact patches. Therefore there will be no movement in the $z$-direction and no rotation about the $x$-axis. Additionally, cornering forces are considered negligible.

Modifying (1) to (7) and then linearizing the result around the operating point $\left(x_{R M}=0 ; \Theta_{P}=0\right.$ and $\delta=$ $0)$ the system's state space equations can be written in matrix form

$$
\begin{aligned}
{\left[\begin{array}{c}
\dot{x}_{R M} \\
\dot{v}_{R M} \\
\dot{\Theta}_{P} \\
\dot{\omega}_{P} \\
\dot{\delta} \\
\ddot{\delta}
\end{array}\right]=} & {\left[\begin{array}{cccccc}
0 & 1 & 0 & 0 & 0 & 0 \\
0 & 0 & A_{23} & 0 & 0 & 0 \\
0 & 0 & 0 & 1 & 0 & 0 \\
0 & 0 & A_{43} & 0 & 0 & 0 \\
0 & 0 & 0 & 0 & 0 & 1 \\
0 & 0 & 0 & 0 & 0 & 0
\end{array}\right]\left[\begin{array}{c}
x_{R M} \\
v_{R M} \\
\Theta_{P} \\
\omega_{P} \\
\delta \\
\dot{\delta}
\end{array}\right] } \\
+ & {\left[\begin{array}{ccccc}
0 & 0 & 0 & 0 & 0 \\
B_{21} & B_{22} & B_{23} & B_{24} & B_{25} \\
0 & 0 & 0 & 0 & 0 \\
B_{41} & B_{42} & B_{43} & B_{44} & B_{45} \\
0 & 0 & 0 & 0 & 0 \\
B_{61} & B_{62} & B_{63} & B_{64} & B_{65}
\end{array}\right]\left[\begin{array}{c}
C_{L} \\
C_{R} \\
f_{d R L} \\
f_{d R R} \\
f_{d P}
\end{array}\right] }
\end{aligned}
$$

where $A_{23}, A_{43}, B_{21}, B_{23}, B_{24}, B_{25}, B_{41}, B_{42}, B_{43}$, $B_{44}, B_{45}, B_{61}, B_{62}, B_{63}, B_{64}$ and $B_{65}$ are defined as a function of the vehicle's parameters.

With this state space model an appropriate control strategy can be developed to keep the vehicle in equilibrium and impose the desired speed and turning rate.

\section{State MeAsuring}

Having developed a dynamic model for JOE, we now need to find appropriate sensors to be able to measure the system's states. These measurements can then be fed back to the controller in order to impose the desired closed loop dynamics.

Straight line position and speed $\left(x_{R M}, v_{R M}\right)$ as well as yaw angle and rate $(\delta, \dot{\delta})$ can be determined from the angle (speed) of rotation of the two wheels with respect to gravity. Fig. 4 shows how these angles $\left(\Theta_{R R}, \Theta_{R L}\right)$ are related to the pitch angle $\Theta_{P}$ and the angle of rotation between the chassis and the wheels $\left(\Theta_{R P R}, \Theta_{R P L}\right)$.

$\Theta_{R P R}$ and $\Theta_{R P L}$ are easily determined with an incremental encoder mounted on each DC motor. Measuring the pitch angle and rate has proven somewhat more difficult. Three potential sensors have been evaluated:

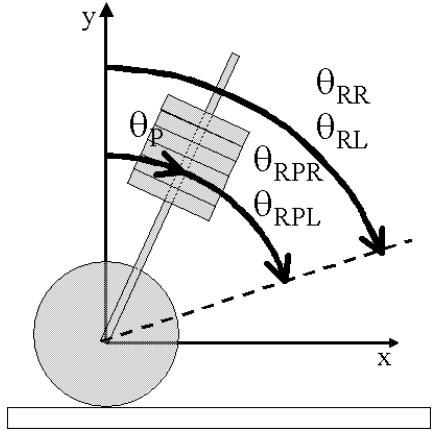

Figure 4. Angles of Rotation

Tilt sensor

measures directly a pitch angle with respect to gravity; differentiation to obtain a pitch rate

Accelerometers measures static and dynamic acceleration; signal decoding in order to obtain a pitch angle; differentiation to obtain a pitch rate

Gyroscope measures a pitch rate; integration in order to obtain a pitch angle

\section{A. Tilt Sensors}

Tilt sensors provide a precise measurement of the pitch angle. Unfortunately, the dynamics of the sensors we evaluated have proven too slow for our application.

\section{B. Accelerometers}

Pitch angle measurement using a combination of two accelerometers has been studied. In such a configuration one of the sensors is mounted on the vehicle to measure acceleration in the y-direction shown on Fig. 5 - the second one measures acceleration in a direction offset by $90^{\circ}$.

Measuring $x_{\text {total }}$ and $y_{\text {total }}$ then allow calculation of the pitch angle $\Theta_{P}$ as well as the unknown acceleration $a$. Simulation as well as tests on the vehicle have shown that those calculations are very sensitive to quantification errors on $x_{\text {total }}$ and $y_{\text {total }}$. A reliable pitch angle measurement can therefore not be achieved.

\section{Gyroscope}

A rate gyroscope directly measures the pitch rate. This signal then needs to be integrated in order to obtain the pitch angle. Due to the gyroscope's characteristics, such an integration is sensitive to drift problems.

This phenomenon can be described by a ramp shaped disturbance angle applied to the system. It can be shown that the chosen control system is able to 


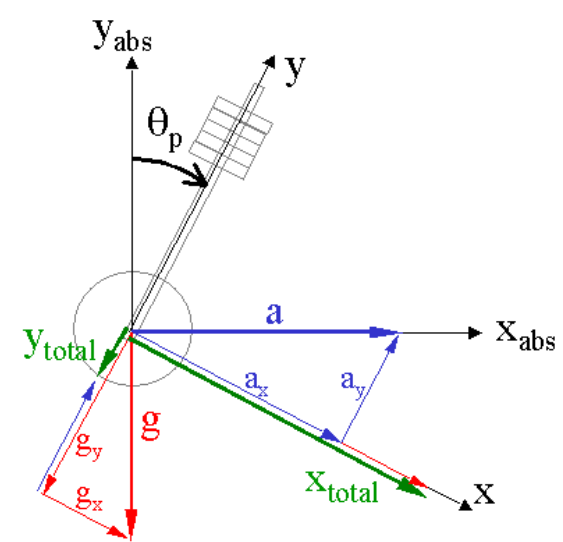

Figure 5. Pitch angle measurement using two accelerometers

reject such a disturbance.

\section{Sensor Integration}

Interfaced as shown on Fig. 6, the two incremental encoders and the rate gyroscope provide information about JOE's states.

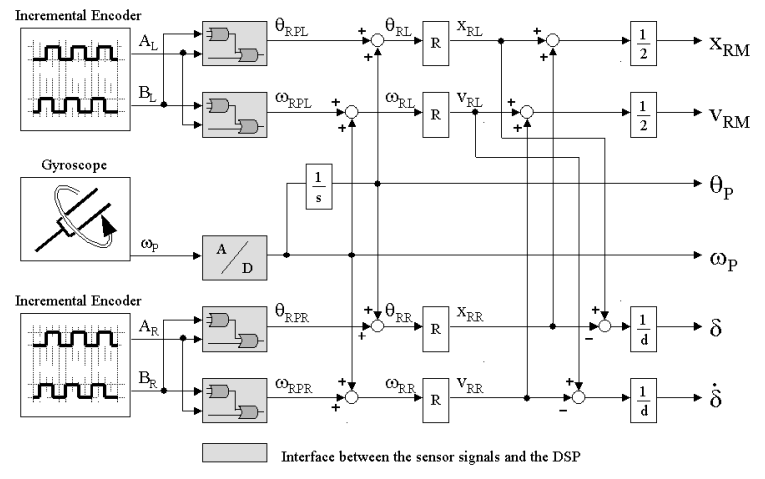

Figure 6. Sensor signal processing

\section{Control System Development}

Having found a way to measure the system's dynamics, we can now focus on the development of the control system, the desired performances and how they can been achieved.

Eq. (8) shows that there are five inputs to our system. Three of those, $f_{d R L}, f_{d R R}$ and $f_{d P}$ are disturbance forces and therefore cannot be used to control the system. However, the torques $C_{L}$ and $C_{R}$ applied to the left and right hand side motor can be governed by the control system.

In order to impose the desired dynamics on the system, we would like to control the rotation around the $z$-axis independently of the rotation around the $y$-axis. This translates into having a controller producing an output signal corresponding to a torque $C_{\delta}$ around the vertical axis and another with an output torque $C_{\Theta}$ around the lateral axis (where each of those torques only influences the dynamics around its axis).

To apply these torques on the system, we need a decoupling unit that transforms $C_{\delta}$ and $C_{\Theta}$ into the wheel torques $C_{L}$ and $C_{R}$. Such a decoupling unit would typically be in the form of:

$$
\left[\begin{array}{l}
C_{L} \\
C_{R}
\end{array}\right]=\left[\begin{array}{ll}
D_{11} & D_{12} \\
D_{21} & D_{22}
\end{array}\right]\left[\begin{array}{l}
C_{\Theta} \\
C_{\delta}
\end{array}\right]
$$

The resulting equation when substituting (9) into (8) needs to be in the following form in order to avoid cross coupling (for the equations to be more legible, the disturbance forces have been omitted):

$$
[\underline{\dot{x}}]=[\underline{A}][\underline{x}]+\left[\begin{array}{cc}
0 & 0 \\
B_{a} & 0 \\
0 & 0 \\
B_{b} & 0 \\
0 & 0 \\
0 & B_{c}
\end{array}\right]\left[\begin{array}{l}
C_{\Theta} \\
C_{\delta}
\end{array}\right]
$$

This can be achieved by choosing:

$$
[\underline{\underline{D}}]=\left[\begin{array}{cc}
0.5 & 0.5 \\
0.5 & -0.5
\end{array}\right]
$$

Note that such a decoupling unit is the electronic equivalent of a mechanical differential as employed in a car or for a robot's wrist where the torque conversion can be written as:

$$
\begin{aligned}
C_{L} & =\frac{1}{2} C_{\Theta}+\frac{1}{2 i} C_{\delta} \\
C_{R} & =\frac{1}{2} C_{\Theta}-\frac{1}{2 i} C_{\delta}
\end{aligned}
$$

Since $[D]$ has been designed for there to be no crosscoupling, the state space equations for the vehicle can now be written as two independent sub-systems: A sub-system "pendulum" describing the rotation about the $z$-axis and a sub-system "rotation" modeling the rotation about the $y$-axis. For the "pendulum" we have:

$$
\left[\begin{array}{c}
\dot{x}_{R M} \\
\dot{v}_{R M} \\
\dot{\Theta}_{P} \\
\dot{\omega}_{P}
\end{array}\right]=\left[\begin{array}{cccc}
0 & 1 & 0 & 0 \\
0 & 0 & A_{23} & 0 \\
0 & 0 & 0 & 1 \\
0 & 0 & A_{43} & 0
\end{array}\right]\left[\begin{array}{c}
x_{R M} \\
v_{R M} \\
\Theta_{P} \\
\omega_{P}
\end{array}\right]+\left[\begin{array}{c}
0 \\
B_{2} \\
0 \\
B_{4}
\end{array}\right]\left[C_{\Theta}\right]
$$

And for the "rotation":

$$
\left[\begin{array}{l}
\dot{\delta} \\
\ddot{\delta}
\end{array}\right]=\left[\begin{array}{ll}
0 & 1 \\
0 & 0
\end{array}\right]\left[\begin{array}{l}
\delta \\
\dot{\delta}
\end{array}\right]+\left[\begin{array}{c}
0 \\
B_{6}
\end{array}\right]\left[C_{\delta}\right]
$$

We are now able to design an independent controller for each of those subsystems with the possibility of assigning different dynamics to each of them.

The design of the state space controllers is straightforward and will not be presented here. We shall, however, come back to the pole placement issue in section 


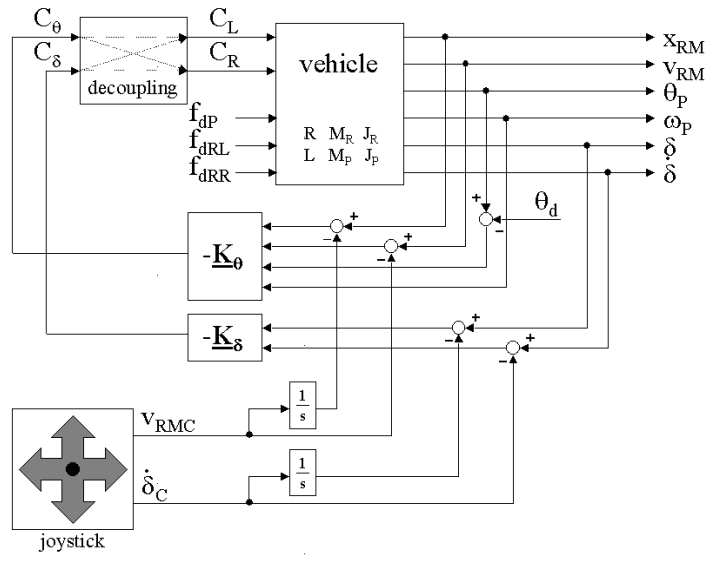

Figure 7. Control system of the vehicle

VI when commenting on the achieved closed loop dynamics.

The user will be able to pilot the system by imposing the straight line speed $v_{R M C}$ of the vehicle as well as its turning rate $\dot{\delta}_{C}$ around the vertical axis (Fig. 7).

\section{Controller Hardware}

The controller has been implemented on a DSP board developed at the LEI and distributed by $C H S$ Engineering (www.chs-eng.ch). It is composed of a Sharc floating point DSP, a XILINX FPGA, 4 10bit $\mathrm{D} / \mathrm{A}$ converters as well as $1412 \mathrm{bit} \mathrm{A} / \mathrm{D}$ converters. A dedicated software enables the user to program the board and get readouts of all the variables while the system is running.

\section{DRIVING the Results}

JOE measures $65 \mathrm{~cm}$ in height and weighs about $12 \mathrm{~kg}$; it can reach a maximum speed of $1.5 \mathrm{~m} / \mathrm{s}$. It is capable of climbing inclines up to $30^{\circ}$ (depending on the actual coefficient of friction). On its $32 \mathrm{~V}, 1.8$ Ah Battery, it has an autonomy of roughly one hour's driving time.

System performance (i.e. reaction to disturbance forces, tracking of driver input, etc.) is driven by the pole placement. In order to maximize JOE's performance, controllers with different pole placements have been tested.

For a chosen pole placement, the controller's gains were calculated and implemented on the DSP board. JOE was then tested with that controller configuration and the response recorded by the control system.

One of the tests conducted consisted of an impulse disturbance force applied to a position above JOE's center of gravity. The energy transmitted with a falling weight amounted to about $1.2 \mathrm{~J}$.

Issues like damping ratio and settling time could be clearly identified on the recorded responses and permitted an efficient fine-tuning of the system.
Fig. 8 shows the system's response to the above mentioned test with the initial pole placement chosen. Note the pronounced oscillation of the system which indicate too weak damping.
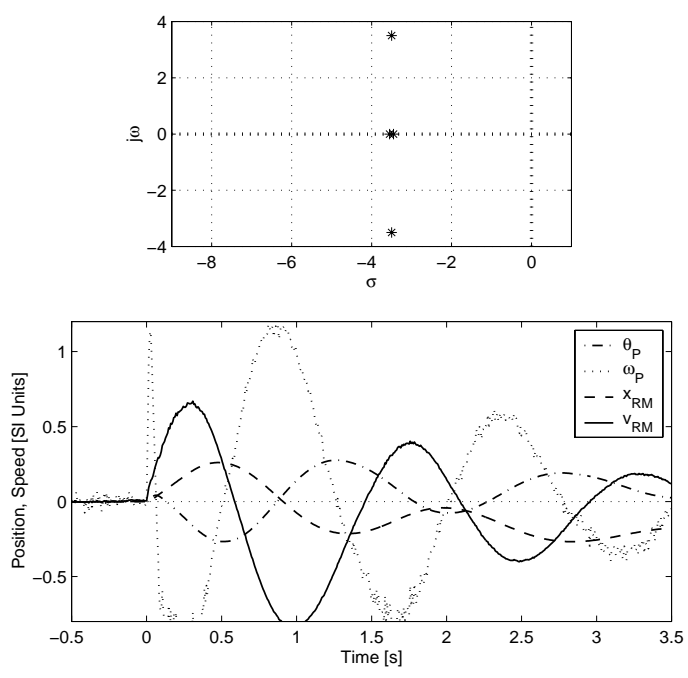

Figure 8. Initial pole placement of the "pendulum" system and associated response to an impulse disturbance force (energy transmitted $\approx 1.2 \mathrm{~J}$ ) applied to the pendulum

Increasing the damping ratio (Fig. 9) improves matters significantly. We now have a harmonious catching of the disturbance force: The weight hitting JOE causes the pendulum to fall forwards $\left(\Theta_{P}>0\right)$. The control system accelerates the wheels in a positive direction to catch this fall and ultimately make the pendulum fall in the other direction. A negative torque is then applied, moving the vehicle back to its original position and getting the pendulum back in an upright position.
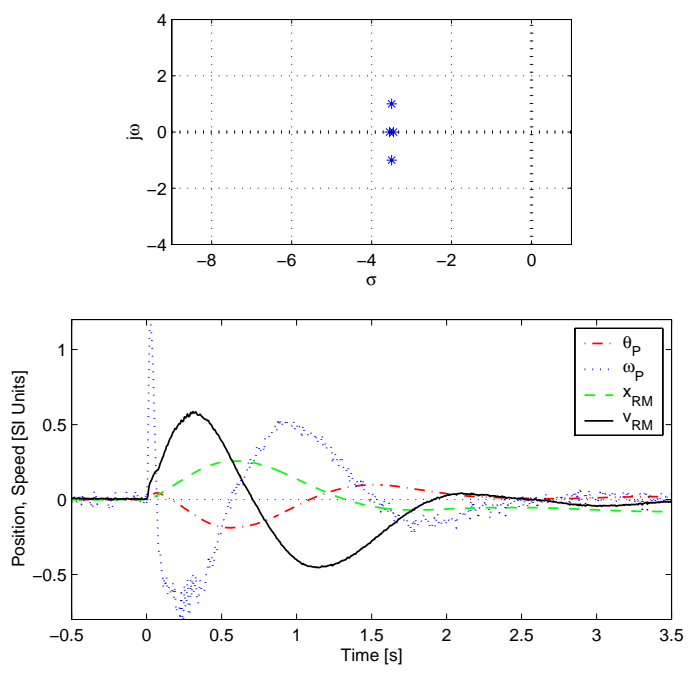

Figure 9. Improved pole placement of the "pendulum" system and associated response to an impulse disturbance force (energy transmitted $\approx 1.2 \mathrm{~J}$ ) applied to the pendulum 
Another issue that has been addressed during testing is driveablility. In order to successfully improve driveablility, it was characterized based on two criteria:

- readouts of the system's reaction to a ramp shaped speed input as well as

- the way different drivers felt about JOE's handling.

Combining the driver's feelings with the readouts of system behaviour allowed further improvement of JOE's control system. Fig. 10 shows the system's response to a velocity ramp input with the final pole placement chosen.

Note that the maximum acceleration possible is lower than the maximum deceleration. Due to the motor's speed-current characteristics, a high torque cannot be obtained when operating at high speeds. However, this is exactly what is necessary to get the vehicle back into an upright position at the end of the acceleration phase. Deceleration demands maximum torque at low speeds - a steeper ramp is therefore possible.
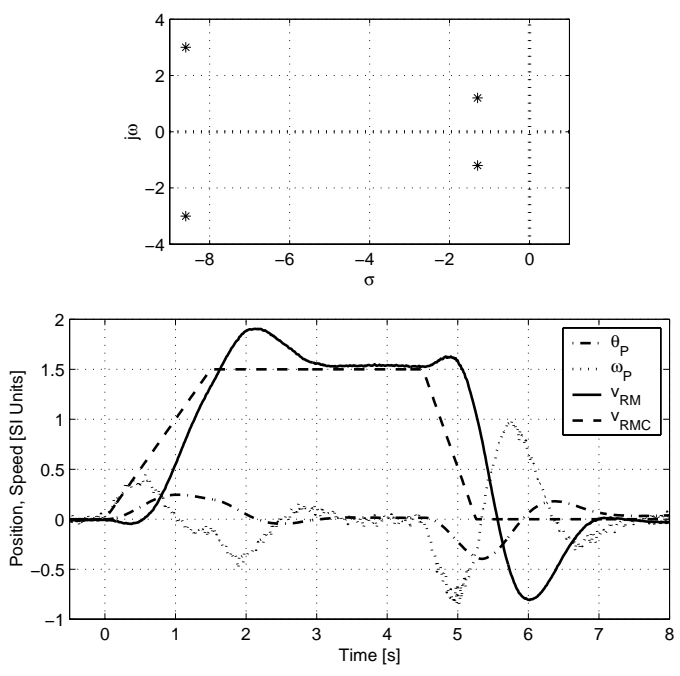

Figure 10. Reaction to a ramp shaped speed input

Increasing performance with the pole placement chosen can be achieved by moving the poles further to the left, thus making the system faster. Backlash (limit cycles) as well the maximum torque that can be transmitted to the ground (grip) prevent us from moving the poles past a certain limit. The authors think that an adaptive pole placement (depending on the system's state) would enable further improvements.

\section{CONCLUSIONS}

This paper has presented a mobile, inverted pendulum. The control system used to guarantee stability of the system is based on two state space controllers, interfaced via a decoupling unit to the two DC motors driving the wheels.

We have shown the performance of the system, its ability to reject force and angular disturbances as well as its capability of tracking a pilot's driving inputs.

A control system varying the pole placement in real time depending on the states and inputs of the system has the potential to further increase JOE's performance. Such an adaptive controller could typically be based on fuzzy logic criteria.

\section{REFERENCES}

[1] D.H. Titterton, J.L. Weston, Strapdown Inertial Navigation Technology, Peter Peregrinus Ltd., 1997.

[2] John H. Mathews, Numerical Methods for Mathematics, Science, and Engineering, Prentice Hall, 1992.

[3] Denis Gillet, Commande Avancée II, Notes de cours, EPFL, 1998.

[4] Roland Longchamp, Commande numérique de systèmes dynamiques, PPUR, 1995.

[5] H. Bühler, Mecatronique, Laboratoire d'électronique industrielle, EPFL, 1992.

[6] S. Colombi, Mecatronique - Notes de cours, Laboratoire d'électronique industrielle, EPFL, 1998.

[7] Marc Nicollerat, Mirko Montemari, Functinal Description of the David Board, CHS-Engineering, 1998.

[8] William F. Riley, Leroy D. Sturges, Engineering Mechanics: Dynamics, John Wiley and Sons, 1996.

[9] A. D'Arrigo, Veicolo elettrico sperimentale a due ruote parallele coassiali indipendenti Tesi di laurea, EPFL/Politecnico di Milano, 1997. 